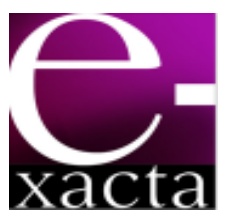

ISSN: 1984-3151

\title{
EFEITO DA TEMPERATURA NA VISCOSIDADE DE ALGUNS ÉSTERES: PREDIÇÃO DA ENERGIA DE ATIVAÇÃO VISCOSA
}

\author{
EFFECT OF TEMPERATURE ON VISCOSITY OF SOME ESTERS: \\ PREDICTION OF ACTIVATION ENERGY FOR VISCOUS FLOW
}

\author{
César Augusto Canciam ${ }^{1}$
}

\begin{abstract}
Mestre em Engenharia Química. UEM. 2001. Professor da Universidade Tecnológica Federal do Paraná - Campus Ponta Grossa - UTFPR. Ponta Grossa, PR. canciam@utfpr.edu.br
\end{abstract}

Recebido em: 24/02/2012 - Aprovado em: 30/06/2012 - Disponibilizado em: 30/07/2012

\begin{abstract}
RESUMO: O conhecimento da viscosidade é um fator de fundamental importância para cálculos que envolvem a seleção de equipamentos e o dimensionamento de bombas e tubulações, assim como para a implementação de um efetivo controle de processos e garantia de qualidade do produto final. O presente trabalho teve por objetivo predizer a energia de ativação viscosa dos ésteres: formato de etila, formato de cis-3-hexenila, acetato de etila, acetato de butila, acetato de isoamila, acetato de hexila, acetato de trans-2-hexenila, acetato de cis-3-hexenila, propionato de etila, butirato de etila, butirato de butila, butirato de isoamila, butirato de hexila, isobutirato de cis-3hexenila, 2-metilbutirato de etila, hexanoato de etila e isovalerato de etila. Para tanto, realizou-se uma análise de regressão linear de dados experimentais da viscosidade de cada éster em função da temperatura. Estes dados experimentais foram encontrados na literatura. Os valores da energia de ativação viscosa para os ésteres estudados encontram-se entre 6,454 e 12,745 $\mathrm{kJ} \mathrm{mol}^{-1}$. Os coeficientes de correlação foram próximos da unidade. A ausência na literatura de valores da energia de ativação viscosa para os ésteres estudados neste trabalho dificultou a comparação e a classificação dos valores da energia de ativação viscosa.
\end{abstract}

PalavRas-Chave: Energia de ativação viscosa. Ésteres. Predição.

ABSTRACT: The knowledge of viscosity is fundamental for calculations that involve the selection of equipments and plotting of pumps and pipes, as well as for the implementation of effective processes control and warranty of quality of the final product. This study aimed to predict the activation energy for viscous flow of esters: ethyl formate, cis-3hexenyl formate, ethyl acetate, butyl acetate, isoamyl acetate, hexyl acetate, trans-2-hexenyl acetate, cis-3-hexenyl acetate, ethyl propionate, ethyl butyrate, butyl butyrate, isoamyl butyrate, hexyl butyrate, cis-3-hexenyl isobutyrate, ethyl 2-methylbutyrate, ethyl hexanoate and ethyl isovalerate. For this purpose, there was a linear regression analysis of experimental data of viscosity of each ester in a function of temperature. These experimental data were found in the literature. The values of activation energy for viscous flow for the esters studied are between 6.454 and $12.745 \mathrm{~kJ} \mathrm{~mol}^{1}$. The correlation coefficients were near to unit. In the literature, the absence of values of activation energy for viscous flow for the esters studied in this work made it difficult to compare and rank the values of activation energy for viscous flow.

KEYWORDS: Activation energy for viscous flow. Esters. Prediction.

\section{INTRODUÇÃO}

A viscosidade é uma propriedade de transporte

fundamental para o projeto de processos nas mais diferentes indústrias químicas e petroquímicas, em que envolvem o transporte de fluidos, agitação, mistura, filtração, concentração e transferência de calor, comentam Lin, Hsieh e Lee (2007). 
A viscosidade de um fluido mede a resistência interna oferecida ao movimento relativo das diferentes partes desse fluido (resistência ao fluxo) (SHAMES, 1999).

Neste sentido, a determinação da viscosidade de líquidos tem como base na Teoria do Estado Ativado de Eyring (1941). Essa teoria considera que um líquido é constituído por moléculas intercaladas por posições desocupadas (vacâncias), e essas moléculas, mesmo com o líquido em repouso, movemse com o propósito de ocuparem as vacâncias adjacentes a elas. A viscosidade está relacionada com a força que tende a se opor a esse movimento, sendo uma medida de fricção interna do fluido. Dessa maneira, quanto maior a barreira potencial que uma molécula terá que vencer a fim de "saltar" para a vacância adjacente, maior é a viscosidade. Essa barreira potencial é conhecida como Energia de Ativação Viscosa (RIBEIRO; DA CRUZ; REIS, 2005; SILVA; PAREDES; REIS, 2007).

A temperatura é um parâmetro relacionado com a energia interna de uma substância. Vários estudos têm demonstrado que a viscosidade de um líquido é altamente influenciada por mudanças na temperatura (OLIVEIRA; BARROS; ROSSI, 2009).

Shames (1999) comenta que a viscosidade é diretamente proporcional à força de atração entre as moléculas. Com o aumento da temperatura, essa força de atração diminui, diminuindo também a viscosidade. Dessa maneira, observa-se nos líquidos que a viscosidade diminui com o aumento da temperatura.

A redução da viscosidade dos líquidos com o aumento da temperatura é atribuída ao aumento das distâncias intermoleculares provocadas durante o aquecimento. $\mathrm{O}$ aumento das distâncias intermoleculares reduz as forças atrativas entre as moléculas, diminuindo a viscosidade (GRANJEIRO et al., 2007).
Giap (2010) comenta que o efeito da temperatura sobre a viscosidade é modelado por uma relação do tipo Arrhenius, conforme a Eq. 1.

$$
\eta=\eta_{\infty} \cdot e^{\left(\frac{E_{a}}{R \cdot T}\right)}
$$

Em que $E_{a}$ corresponde à Energia de Ativação Viscosa; $R$, à constante dos gases ideais; $T$, à temperatura absoluta; $\eta$, à viscosidade e $\eta_{\infty}$, à viscosidade quando a temperatura tende ao infinito.

De acordo com Gratão, Berto e Silveira Júnior (2004), a Eq. 1 pode ser reescrita na forma de:

$$
\ln \eta=\ln \eta_{\infty}+\left(\frac{E_{a}}{R}\right) \cdot \frac{1}{T}
$$

Um gráfico de $\ln \eta$ versus $\frac{1}{T}$, segundo Canciam (2008), fornece uma reta, em que o coeficiente angular é numericamente igual à razão $\left(\frac{E_{a}}{R}\right)$ e o coeficiente linear, à $\ln \eta_{\infty}$.

A Eq. 2 indica a dependência da viscosidade de um fluido com a temperatura e a mesma pode ser utilizada com dados experimentais da viscosidade, na determinação da Energia de Ativação Viscosa e do parâmetro $\eta_{\infty}$. O conhecimento destes parâmetros permite verificar os efeitos da temperatura sobre a viscosidade, além de modelar matematicamente o comportamento reológico desse fluido (GRATÃO; BERTO; SILVEIRA JÚNIOR, 2004).

Com relação à Energia de Ativação Viscosa $\left(E_{a}\right)$, esta grandeza indica a sensibilidade da viscosidade devido à mudança de temperatura, de maneira que, quanto maior for a Energia de Ativação Viscosa, maior será a influência da temperatura, ou seja, alta Energia de Ativação Viscosa indica uma mudança mais rápida 
na viscosidade com a temperatura (PEREIRA; QUEIROZ; FIGUEIRÊDO, 2003; SILVA, 2008).

Os ésteres são compostos orgânicos derivados dos ácidos carboxílicos pela substituição da sua hidroxila por um grupo alquila (proveniente de álcoois) ou arila (proveniente de fenóis). São substâncias, via de regra, líquidas, menos densas que a água e que apresentam ponto de ebulição normal, devido à ausência de ligações de hidrogênio (CAMPOS et al., 1997; SKORONSKI, 2006).

Devido à presença de dois átomos de oxigênio e ao ângulo entre as ligações dos átomos no grupo funcional, as moléculas dos ésteres apresentam certa polaridade. Essa polaridade é mais acentuada em ésteres de cadeia carbônica pequena (massa molecular pequena), em que predominam as interações intermoleculares do tipo dipolo permanente-dipolo permanente. À medida que a massa molecular dos ésteres aumenta, a polaridade vai se tornando menor e passam a existir interações intermoleculares do tipo dipolo instantâneo-dipolo induzido ou também chamadas de forças de Van der Waals (CAMPOS et al., 1997; SOLOMONS e FRYHLE, 2009).

Os ésteres de menor massa molecular são líquidos, conforme aumenta a massa molecular passam de líquidos oleosos e viscosos a sólidos (SOLOMONS e FRYHLE, 2009).

Ésteres de menor massa molecular são parcialmente solúveis em água. Demais ésteres são insolúveis em água. Ésteres de maior massa molecular são solúveis em muitos solventes orgânicos apolares e pouco polares como hidrocarbonetos aromáticos, haletos de alquila e éteres, devido às interações intermoleculares do tipo dipolo instantâneo-dipolo induzido. São solúveis em álcoois e os ésteres com até três átomos de carbono são praticamente solúveis em água (SOLOMONS e FRYHLE, 2009; SKORONSKI, 2006).
Os ésteres são frequentemente empregados como solventes em reações orgânicas porque formam um meio reacional moderadamente polar e aprótico. É por esse motivo que os ésteres são utilizados como solventes em diversos setores industriais como petroquímica, alimentos, cosméticos, tintas e vernizes, entre outros (SKORONSKI, 2006).

Destaca-se também a utilização dos ésteres como plastificantes e secantes. Os ésteres são encontrados de maneira natural em condimentos, flores e frutas (COSTA et al., 2004; GARCIA, 2008).

Um grande número de ésteres são voláteis e apresentam odor geralmente agradável, sendo que alguns deles são utilizados na indústria de perfumes ou na formulação de aromas empregados nos alimentos (BRUICE, 2006).

O objetivo deste trabalho foi predizer a Energia de Ativação Viscosa para os ésteres: formato de etila, formato de cis-3-hexenila, acetato de etila, acetato de butila, acetato de isoamila, acetato de hexila, acetato de trans-2-hexenila, acetato de cis-3-hexenila, propionato de etila, butirato de etila, butirato de butila, butirato de isoamila, butirato de hexila, isobutirato de cis-3-hexenila, 2-metilbutirato de etila, hexanoato de etila e isovalerato de etila. A predição da Energia de Ativação Viscosa utilizou a regressão linear dos dados da viscosidade de cada um dos ésteres em função da temperatura, encontrados em outro trabalho.

\section{Metodologia}

O cálculo da razão $\frac{E_{a}}{R}$, de $\ln \eta_{\infty}$ e do coeficiente de correlação $\left(r^{2}\right)$ seguiu a rotina indicada pela FIG. 1.

A Eq. 3 e a Eq. 8 são adaptações do trabalho de Triola (2008) e determinam, respectivamente, a razão $\frac{E_{a}}{R} \mathrm{e}$ $\ln \eta_{\infty}$ 


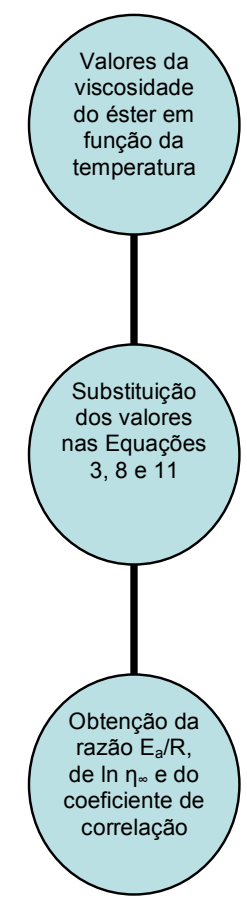

Figura 1 - Rotina de cálculo para a obtenção da razão $\frac{E_{a}}{R}$, de $\ln \eta_{\infty}$ e do coeficiente de correlação de cada um dos ésteres estudados

$$
\frac{E_{a}}{R}=\frac{(n \cdot c)-d}{(n \cdot f)-g}
$$

Em que:

$$
\begin{array}{r}
c=\sum\left(\frac{1}{T} \cdot \ln \eta\right) \\
d=\left(\sum \frac{1}{T}\right) \cdot \sum \ln \eta \\
f=\sum\left(\frac{1}{T}\right)^{2} \\
g=\left(\sum \frac{1}{T}\right)^{2} \\
\ln \eta_{\infty}=\frac{(n \cdot f)}{(n \cdot h)-(d \cdot i)-g}
\end{array}
$$

Em que:

Disponível em: www.unibh.br/revistas/exacta/

$$
\begin{gathered}
h=\sum \ln \eta \\
i=\sum \frac{1}{T}
\end{gathered}
$$

A Eq. 11, também adaptada de Triola (2008), fornece o coeficiente de correlação $\left(r^{2}\right)$ para a análise de regressão linear dos dados de $\ln \eta$ versus $\frac{1}{T}$.

$$
r^{2}=\frac{(n \cdot c)-d}{\sqrt{[(n \cdot f)-g] \cdot\left[(n \cdot j)-h^{2}\right]}}
$$

Em que:

$$
j=\sum(\ln \eta)^{2}
$$

Sendo $n$ correspondente ao número de dados amostrais emparelhados.

O produto da multiplicação da razão $\frac{E_{a}}{R}$ por $R$, fornece como resultado a Energia de Ativação Viscosa $\left(E_{a}\right)$. Para este cálculo, foi considerado que a constante dos gases ideais $(R)$ é igual a $8,314 \times 10^{-3}$ $\mathrm{kJ} \cdot \mathrm{mol}^{-1} \cdot \mathrm{K}^{-1}$.

\section{Resultados e discussão}

A TAB. 1, a TAB. 2, a TAB. 3 e a TAB. 4 indicam, respectivamente, os dados da viscosidade $(\eta)$ do formato de etila $\left(\mathrm{C}_{3} \mathrm{H}_{6} \mathrm{O}_{2}\right)$, do formato de cis-3-hexenila $\left(\mathrm{C}_{7} \mathrm{H}_{12} \mathrm{O}_{2}\right)$, do acetato de etila $\left(\mathrm{C}_{4} \mathrm{H}_{8} \mathrm{O}_{2}\right)$ e do acetato de butila $\left(\mathrm{C}_{6} \mathrm{H}_{12} \mathrm{O}_{2}\right)$ em função da temperatura $(T)$. Estes dados foram obtidos de Djojoputro e Ismadji (2005).

A TAB. 5, a TAB. 6, a TAB. 7 e a TAB.8 relacionam, respectivamente, os dados da viscosidade dos acetatos de isoamila $\left(\mathrm{C}_{7} \mathrm{H}_{14} \mathrm{O}_{2}\right)$, hexila $\left(\mathrm{C}_{8} \mathrm{H}_{16} \mathrm{O}_{2}\right)$, 
trans-2-hexenila $\left(\mathrm{C}_{8} \mathrm{H}_{14} \mathrm{O}_{2}\right)$ e cis-3-hexenila $\left(\mathrm{C}_{8} \mathrm{H}_{14} \mathrm{O}_{2}\right)$ em função da temperatura. Estes dados foram obtidos de Djojoputro e Ismadji (2005).

Tabela 1

Viscosidade do formato de etila em função da temperatura

\begin{tabular}{c|c}
\hline Temperatura (K) & Viscosidade (mPa.s) \\
\hline 293,15 & 0,402 \\
298,15 & 0,381 \\
303,15 & 0,362 \\
308,15 & 0,345 \\
313,15 & 0,329 \\
318,15 & 0,314 \\
323,15 & 0,300 \\
\hline \multicolumn{2}{|c|}{}
\end{tabular}

Tabela 2

Viscosidade do formato de cis-3-hexenila em função da temperatura

\begin{tabular}{c|c}
\hline Temperatura (K) & Viscosidade (mPa.s) \\
\hline 293,15 & 0,599 \\
298,15 & 0,561 \\
303,15 & 0,527 \\
308,15 & 0,496 \\
313,15 & 0,469 \\
318,15 & 0,443 \\
323,15 & 0,421 \\
328,15 & 0,400 \\
333,15 & 0,380 \\
338,15 & 0,363 \\
343,15 & 0,346 \\
\hline
\end{tabular}

A TAB. 9 indica os dados da viscosidade do propionato de etila $\left(\mathrm{C}_{5} \mathrm{H}_{10} \mathrm{O}_{2}\right)$ em função da temperatura. Estes dados foram obtidos de Djojoputro e Ismadji (2005).

A TAB. 10 e a TAB. 11 relacionam, respectivamente, os dados da viscosidade dos butiratos de etila $\left(\mathrm{C}_{6} \mathrm{H}_{12} \mathrm{O}_{2}\right)$ e butila $\left(\mathrm{C}_{8} \mathrm{H}_{16} \mathrm{O}_{2}\right)$ em função da temperatura. Estes dados foram obtidos de Djojoputro e Ismadji (2005).

Tabela 3

Viscosidade do acetato de etila em função da temperatura

\begin{tabular}{c|c}
\hline Temperatura (K) & Viscosidade (mPa.s) \\
\hline 293,15 & 0,443 \\
298,15 & 0,421 \\
303,15 & 0,399 \\
308,15 & 0,381 \\
313,15 & 0,363 \\
318,15 & 0,346 \\
323,15 & 0,330 \\
328,15 & 0,315 \\
333,15 & 0,301 \\
338,15 & 0,288 \\
343,15 & 0,275 \\
\hline
\end{tabular}

Tabela 4

Viscosidade do acetato de butila em função da temperatura

\begin{tabular}{c|c}
\hline Temperatura (K) & Viscosidade (mPa.s) \\
\hline 293,15 & 0,732 \\
298,15 & 0,677 \\
303,15 & 0,628 \\
308,15 & 0,585 \\
313,15 & 0,546 \\
318,15 & 0,512 \\
323,15 & 0,481 \\
328,15 & 0,453 \\
333,15 & 0,428 \\
338,15 & 0,405 \\
343,15 & 0,384 \\
\hline
\end{tabular}

Fonte - DJOJOPUTRO; ISMADJI, 2005, p. 729.

A TAB. 12 e a TAB. 13 relacionam, respectivamente, os dados da viscosidade dos butiratos de isoamila $\left(\mathrm{C}_{9} \mathrm{H}_{18} \mathrm{O}_{2}\right)$ e hexila $\left(\mathrm{C}_{10} \mathrm{H}_{20} \mathrm{O}_{2}\right)$ em função da temperatura. Estes dados foram obtidos de Djojoputro e Ismadji (2005). 
Tabela 5

Viscosidade do acetato de isoamila em função da temperatura

\begin{tabular}{c|c}
\hline Temperatura (K) & Viscosidade (mPa.s) \\
\hline 293,15 & 0,888 \\
298,15 & 0,827 \\
303,15 & 0,773 \\
308,15 & 0,724 \\
313,15 & 0,678 \\
318,15 & 0,638 \\
323,15 & 0,601 \\
328,15 & 0,567 \\
333,15 & 0,536 \\
338,15 & 0,507 \\
343,15 & 0,481 \\
\hline
\end{tabular}

Fonte - DJOJOPUTRO; ISMADJI, 2005, p. 729.

Tabela 6

Viscosidade do acetato de hexila em função da temperatura

\begin{tabular}{c|c}
\hline Temperatura (K) & Viscosidade (mPa.s) \\
\hline 293,15 & 1,118 \\
298,15 & 1,036 \\
303,15 & 0,962 \\
308,15 & 0,895 \\
313,15 & 0,836 \\
318,15 & 0,782 \\
323,15 & 0,733 \\
328,15 & 0,689 \\
333,15 & 0,649 \\
338,15 & 0,612 \\
343,15 & 0,579 \\
\hline
\end{tabular}

Fonte - DJOJOPUTRO; ISMADJI, 2005, p. 729.

A TAB. 14 e a TAB. 15 indicam, respectivamente, os dados da viscosidade do isobutirato de cis-3-hexenila $\left(\mathrm{C}_{10} \mathrm{H}_{18} \mathrm{O}_{2}\right)$ e do 2-metilbutirato de etila $\left(\mathrm{C}_{7} \mathrm{H}_{14} \mathrm{O}_{2}\right)$ em função da temperatura. Estes dados foram obtidos de Djojoputro e Ismadji (2005).

A TAB. 16 e a TAB. 17 indicam, respectivamente, os dados da viscosidade do hexanoato de etila $\left(\mathrm{C}_{8} \mathrm{H}_{16} \mathrm{O}_{2}\right)$ e do isovalerato de etila $\left(\mathrm{C}_{7} \mathrm{H}_{14} \mathrm{O}_{2}\right)$ em função da temperatura. Estes dados foram obtidos de Djojoputro e Ismadji (2005).
Tabela 7

Viscosidade do acetato de trans-2-hexenila em função da temperatura

\begin{tabular}{c|c}
\hline Temperatura (K) & Viscosidade (mPa.s) \\
\hline 293,15 & 0,901 \\
298,15 & 0,831 \\
303,15 & 0,770 \\
308,15 & 0,715 \\
313,15 & 0,666 \\
318,15 & 0,622 \\
323,15 & 0,582 \\
328,15 & 0,547 \\
333,15 & 0,514 \\
\hline
\end{tabular}

Fonte - DJOJOPUTRO; ISMADJI, 2005, p. 729.

Tabela 8

Viscosidade do acetato de cis-3-hexenila em função da temperatura

\begin{tabular}{c|c}
\hline Temperatura (K) & Viscosidade (mPa.s) \\
\hline 293,15 & 0,893 \\
298,15 & 0,823 \\
303,15 & 0,763 \\
308,15 & 0,708 \\
313,15 & 0,660 \\
318,15 & 0,616 \\
323,15 & 0,576 \\
328,15 & 0,542 \\
333,15 & 0,509 \\
\hline
\end{tabular}

Tabela 9

Viscosidade do propionato de etila em função da temperatura

\begin{tabular}{c|c}
\hline Temperatura (K) & Viscosidade (mPa.s) \\
\hline 293,15 & 0,523 \\
298,15 & 0,492 \\
303,15 & 0,464 \\
308,15 & 0,441 \\
313,15 & 0,418 \\
318,15 & 0,398 \\
323,15 & 0,378 \\
328,15 & 0,360 \\
333,15 & 0,344 \\
338,15 & 0,329 \\
343,15 & 0,315 \\
\hline
\end{tabular}

Fonte - DJOJOPUTRO; ISMADJI, 2005, p. 729. 
Tabela 10

Viscosidade do butirato de etila em função da temperatura

\begin{tabular}{c|c}
\hline Temperatura (K) & Viscosidade (mPa.s) \\
\hline 293,15 & 0,662 \\
298,15 & 0,620 \\
303,15 & 0,583 \\
308,15 & 0,549 \\
313,15 & 0,519 \\
318,15 & 0,491 \\
323,15 & 0,465 \\
328,15 & 0,442 \\
333,15 & 0,420 \\
338,15 & 0,400 \\
343,15 & 0,382 \\
\hline
\end{tabular}

Fonte - DJOJOPUTRO; ISMADJI, 2005, p. 729.

Tabela 11

Viscosidade do butirato de butila em função da temperatura

\begin{tabular}{c|c}
\hline Temperatura $(\mathrm{K})$ & Viscosidade (mPa.s) \\
\hline 293,15 & 0,948 \\
298,15 & 0,876 \\
303,15 & 0,812 \\
308,15 & 0,755 \\
313,15 & 0,705 \\
318,15 & 0,659 \\
323,15 & 0,618 \\
328,15 & 0,581 \\
333,15 & 0,548 \\
338,15 & 0,517 \\
343,15 & 0,486 \\
\hline
\end{tabular}

Fonte - DJOJOPUTRO; ISMADJI, 2005, p. 729.
Tabela 12

Viscosidade do butirato de isoamila em função da temperatura

\begin{tabular}{c|c}
\hline Temperatura $(\mathrm{K})$ & Viscosidade (mPa.s) \\
\hline 293,15 & 1,044 \\
298,15 & 0,967 \\
303,15 & 0,897 \\
308,15 & 0,836 \\
313,15 & 0,781 \\
318,15 & 0,731 \\
323,15 & 0,686 \\
328,15 & 0,646 \\
333,15 & 0,609 \\
338,15 & 0,575 \\
343,15 & 0,545 \\
\hline
\end{tabular}

Fonte - DJOJOPUTRO; ISMADJI, 2005, p. 729.

Tabela 13

Viscosidade do butirato de hexila em função da temperatura

\begin{tabular}{c|c}
\hline Temperatura (K) & Viscosidade (mPa.s) \\
\hline 293,15 & 1,351 \\
298,15 & 1,234 \\
303,15 & 1,131 \\
308,15 & 1,014 \\
313,15 & 0,961 \\
318,15 & 0,889 \\
323,15 & 0,825 \\
328,15 & 0,768 \\
333,15 & 0,718 \\
338,15 & 0,669 \\
343,15 & 0,627 \\
\hline
\end{tabular}

Fonte-DJOJOPUTRO; ISMADJI, 2005, p. 730. 
Tabela 14

Viscosidade do isobutirato de cis-3-hexenila em função da temperatura

\begin{tabular}{c|c}
\hline Temperatura (K) & Viscosidade (mPa.s) \\
\hline 293,15 & 1,024 \\
298,15 & 0,941 \\
303,15 & 0,868 \\
308,15 & 0,804 \\
313,15 & 0,741 \\
318,15 & 0,690 \\
323,15 & 0,650 \\
328,15 & 0,606 \\
333,15 & 0,560 \\
338,15 & 0,535 \\
343,15 & 0,503 \\
\hline
\end{tabular}

Fonte - DJOJOPUTRO; ISMADJI, 2005, p. 730.

Tabela 15

Viscosidade do 2-metilbutirato de etila em função da temperatura

\begin{tabular}{c|c}
\hline Temperatura (K) & Viscosidade (mPa.s) \\
\hline 293,15 & 0,831 \\
298,15 & 0,774 \\
303,15 & 0,723 \\
308,15 & 0,677 \\
313,15 & 0,635 \\
318,15 & 0,597 \\
323,15 & 0,563 \\
328,15 & 0,532 \\
333,15 & 0,503 \\
338,15 & 0,476 \\
343,15 & 0,452 \\
\hline
\end{tabular}

Fonte - DJOJOPUTRO; ISMADJI, 2005, p. 730.

A partir dos dados da viscosidade $(\eta)$ em função da temperatura $(T)$, foi plotado o gráfico de $\ln \eta$ versus $\frac{1}{T}$ para cada éster estudado.

A FIG. 2 ilustra o gráfico de $\ln \eta$ versus $\frac{1}{T}$ para os formatos de etila e cis-3-hexenila.
Tabela 16

Viscosidade do hexanoato de etila em função da temperatura

\begin{tabular}{c|c}
\hline Temperatura (K) & Viscosidade (mPa.s) \\
\hline 293,15 & 0,816 \\
298,15 & 0,757 \\
303,15 & 0,704 \\
308,15 & 0,656 \\
313,15 & 0,614 \\
318,15 & 0,576 \\
323,15 & 0,541 \\
328,15 & 0,509 \\
333,15 & 0,482 \\
338,15 & 0,456 \\
343,15 & 0,429 \\
\hline
\end{tabular}

Fonte - DJOJOPUTRO; ISMADJI, 2005, p. 730.

Tabela 17

Viscosidade do isovalerato de etila em função da temperatura

\begin{tabular}{c|c}
\hline Temperatura (K) & Viscosidade (mPa.s) \\
\hline 293,15 & 0,805 \\
298,15 & 0,752 \\
303,15 & 0,703 \\
308,15 & 0,659 \\
313,15 & 0,620 \\
318,15 & 0,581 \\
323,15 & 0,544 \\
328,15 & 0,516 \\
333,15 & 0,485 \\
338,15 & 0,457 \\
343,15 & 0,431 \\
\hline
\end{tabular}

Fonte - DJOJOPUTRO; ISMADJI, 2005, p. 730.

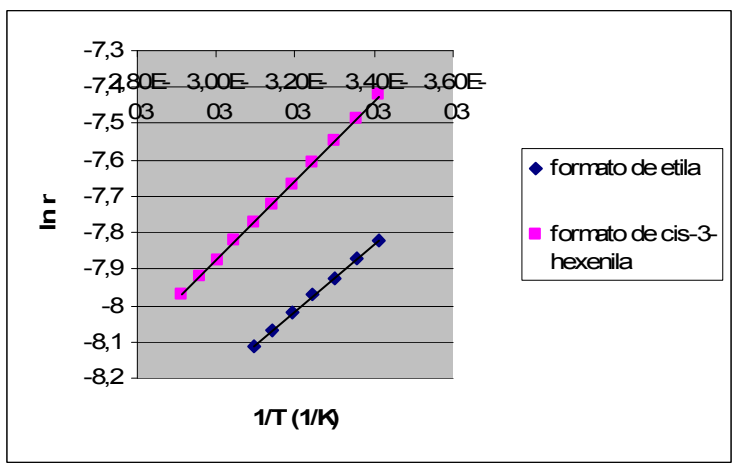

Figura 2 - Gráfico de $\ln \eta$ versus $\frac{1}{T}$ para os formatos de etila e cis-3-hexenila 
A FIG. 3 ilustra o gráfico de $\ln \eta$ versus $\frac{1}{T}$ para os acetatos de etila, butila, isoamila e hexila.

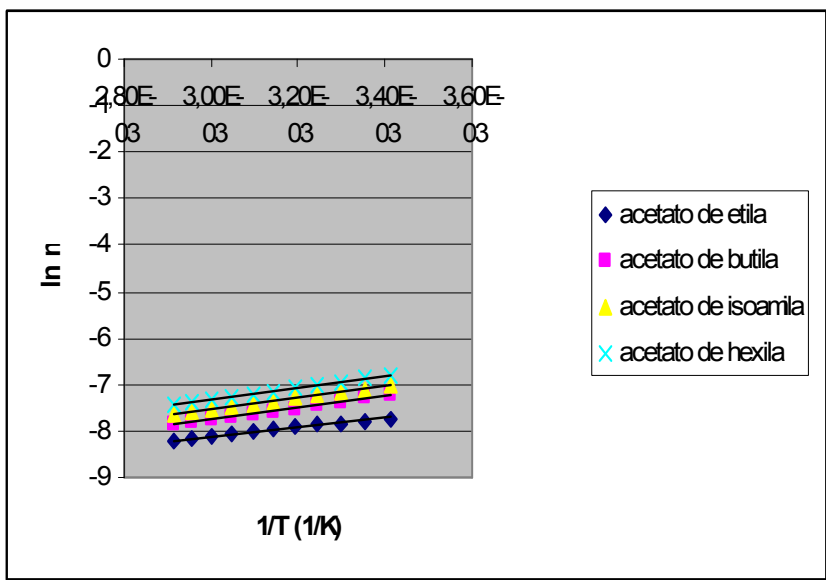

Figura 3 - Gráfico de $\ln \eta$ versus $\frac{1}{T}$ para os acetatos de etila, butila, isoamila e hexila

A FIG. 4 ilustra o gráfico de $\ln \eta$ versus $\frac{1}{T}$ para o acetato de trans-2-hexenila, propionato de etila e hexanoato de etila.

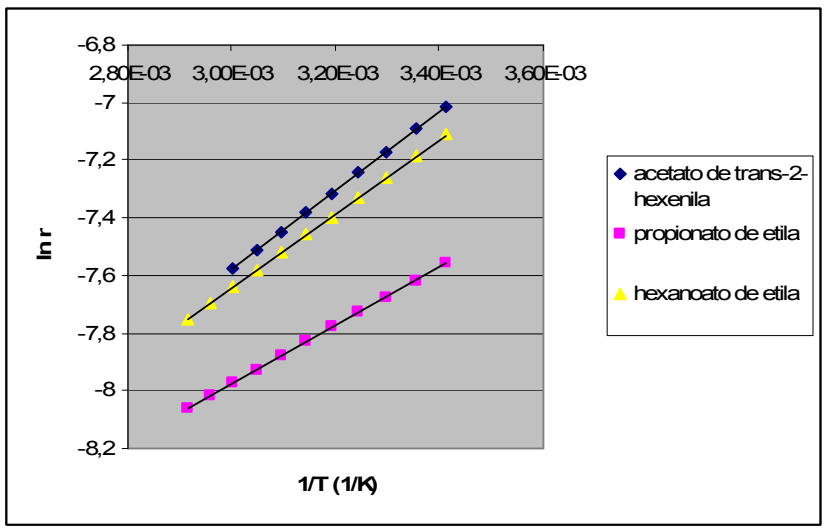

Figura 4 - Gráfico de $\ln \eta$ versus $\frac{1}{T}$ para o acetato de trans-2-hexenila, propionato de etila e hexanoato de etila
A FIG. 5 ilustra o gráfico de $\ln \eta$ versus $\frac{1}{T}$ para o acetato de cis-3-hexenila, isobutirato de cis-3hexenila, isovalerato de etila e 2-metilbutirato de etila.

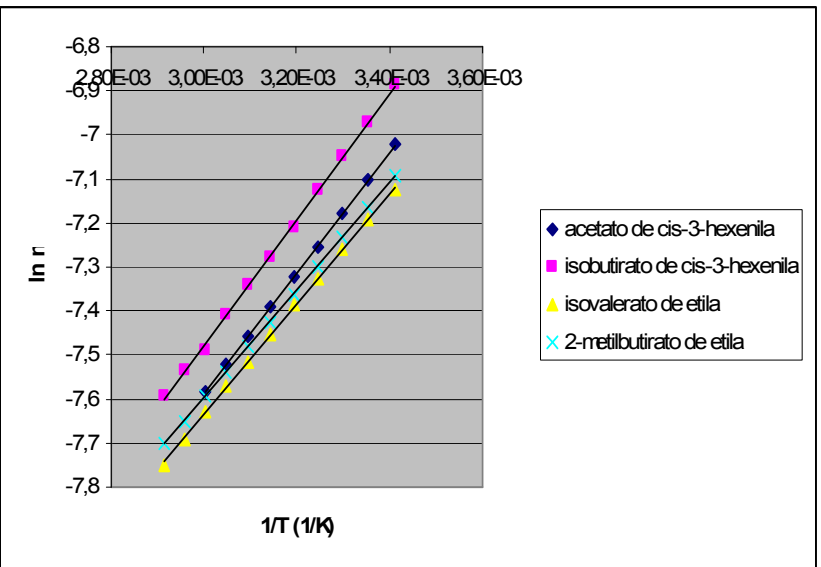

Figura 5 - Gráfico de $\ln \eta$ versus $\frac{1}{T}$ para o acetato de cis-3-hexenila, isobutirato de cis-3-hexenila, isovalerato de etila e 2-metilbutirato de etila

A FIG. 6 ilustra o gráfico de $\ln \eta$ versus $\frac{1}{T}$ para os butiratos de etila, butila, isoamila e hexila.

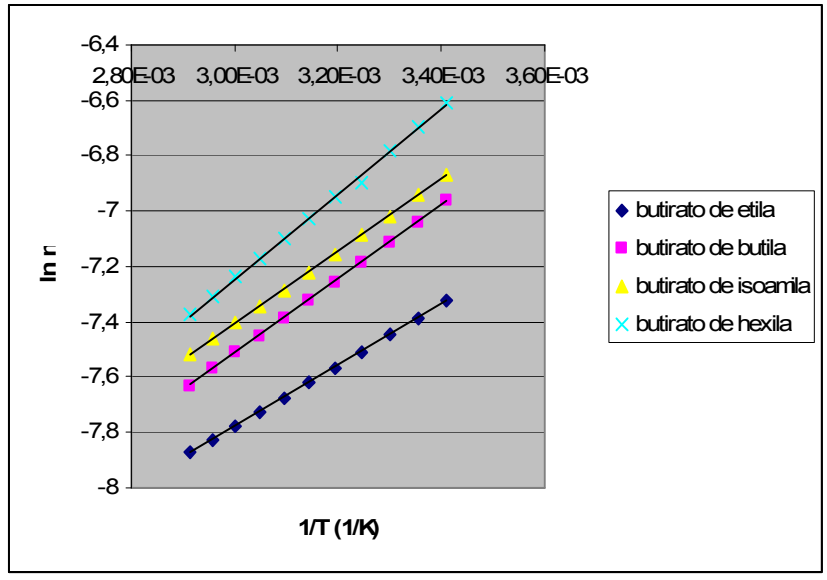

Figura 6 - Gráfico de $\ln \eta$ versus $\frac{1}{T}$ para os butiratos de etila, butila, isoamila e hexila 
A TAB. 18 relaciona a massa molecular dos ésteres estudados.

\section{Tabela 18}

Massa molecular dos ésteres estudados

\begin{tabular}{c|c}
\hline Éster & $\begin{array}{c}\text { Massa molecular } \\
\text { (u.m.a.) }\end{array}$ \\
\hline Formato de etila & 74,08 \\
Formato de cis-3-hexenila & 128,17 \\
Acetato de etila & 88,11 \\
Acetato de butila & 116,16 \\
Acetato de isoamila & 130,19 \\
Acetato de hexila & 144,21 \\
Acetato de trans-2- & 142,20 \\
hexenila & 142,20 \\
Acetato de cis-3-hexenila & 102,13 \\
Propionato de etila & 116,16 \\
Butirato de etila & 144,21 \\
Butirato de butila & 158,23 \\
Butirato de isoamila & 172,27 \\
Butirato de hexila & 170,25 \\
Isobutirato de cis-3- & \\
hexenila & 130,19 \\
Isovalerato de etila & 130,19 \\
2-metilbutirato de etila & 144,21 \\
Hexanoato de etila & \\
\hline Fon & \\
\hline
\end{tabular}

Fonte - DJOJOPUTRO; ISMADJI, 2005, p. 728.

A TAB. 19 relaciona os valores encontrados para a razão $\frac{E_{a}}{R}$. Enquanto que a TAB. 20 , os valores encontrados para $\ln \eta_{\infty}$.

O coeficiente de correlação $\left(r^{2}\right)$, determinado através da Eq. 11, obteve valor igual a 0,999 para todos os ésteres, com exceção do acetato de etila, que apresentou coeficiente de correlação igual a 0,962.

De acordo com Triola (2008), o coeficiente de correlação mede o grau de relacionamento linear entre os valores emparelhados das variáveis dependente e independente em uma amostra, avaliando assim, a "qualidade" do ajuste.
Tabela 19

Valores encontrados para $\frac{E_{a}}{R}$

\begin{tabular}{c|c}
\hline Éster & $\frac{E_{a}}{R}(\mathrm{~K})$ \\
\hline Formato de etila & 922,038 \\
\hline Formato de cis-3-hexenila & 1100,536 \\
Acetato de etila & 776,308 \\
Acetato de butila & 1296,146 \\
Acetato de isoamila & 1232,637 \\
Acetato de hexila & 1324,966 \\
Acetato de trans-2- & 1369,130 \\
hexenila & 1369,761 \\
Acetato de cis-3-hexenila & 1016,788 \\
Propionato de etila & 1105,066 \\
Butirato de etila & 1335,522 \\
Butirato de butila & 1308,189 \\
Butirato de isoamila & 1533,003 \\
Butirato de hexila & 1434,538 \\
Isobutirato de cis-3- & \\
hexenila & 1252,901 \\
Isovalerato de etila & 1224,067 \\
2-metilbutirato de etila & 1285,429 \\
Hexanoato de etila & \\
\hline
\end{tabular}

Tabela 20

Valores encontrados para $\ln \eta_{\infty}$

\begin{tabular}{c|c}
\hline Éster & $\ln \eta_{\infty}$ \\
\hline Formato de etila & $-10,965$ \\
Formato de cis-3-hexenila & $-11,178$ \\
Acetato de etila & $-10,425$ \\
Acetato de butila & $-11,648$ \\
Acetato de isoamila & $-11,232$ \\
Acetato de hexila & $-11,317$ \\
Acetato de trans-2- & $-11,685$ \\
hexenila & $-11,696$ \\
Acetato de cis-3-hexenila & $-11,027$ \\
Propionato de etila & $-11,092$ \\
Butirato de etila & $-11,520$ \\
Butirato de butila & $-11,331$ \\
Butirato de isoamila & $-11,844$ \\
Butirato de hexila & $-11,782$ \\
Isobutirato de cis-3- & $-11,393$ \\
hexenila & $-11,270$ \\
Isovalerato de etila & $-11,499$ \\
2-metilbutirato de etila & \\
Hexanoato de etila & \\
\hline
\end{tabular}


Triola (2008) comenta que o coeficiente de correlação quanto mais próximo for da unidade, melhor é a "qualidade" do ajuste da função aos pontos do diagrama de dispersão.

Callegari-Jaques (2003), citado por Lira (2004), comenta que para valores do coeficiente de correlação $\left(r^{2}\right)$ maiores ou iguais a 0,90 e menores que 1,0 , a correlação linear é classificada como muito forte. Dessa forma, para todos os ésteres estudados, a correlação linear é classificada como muito forte.

A TAB. 21 indica os valores encontrados para a Energia de Ativação Viscosa $\left(E_{a}\right)$.

Tabela 21

Valores encontrados para a Energia de Ativação Viscosa

\begin{tabular}{c|c}
\hline Éster & $E_{a}\left(\mathrm{~kJ}^{\mathrm{mol}}{ }^{-1}\right)$ \\
& \\
\hline Formato de etila & 7,669 \\
Formato de cis-3-hexenila & 9,150 \\
Acetato de etila & 6,454 \\
Acetato de butila & 10,776 \\
Acetato de isoamila & 10,248 \\
Acetato de hexila & 11,016 \\
Acetato de trans-2- & 11,383 \\
hexenila & 11,388 \\
Acetato de cis-3-hexenila & 8,454 \\
Propionato de etila & 9,188 \\
Butirato de etila & 11,104 \\
Butirato de butila & 10,876 \\
Butirato de isoamila & 12,745 \\
Butirato de hexila & 11,927 \\
Isobutirato de cis-3- & \\
hexenila & 10,417 \\
Isovalerato de etila & 10,177 \\
2-metilbutirato de etila & 10,687 \\
Hexanoato de etila & \\
\hline
\end{tabular}

Pode-se observar na TAB. 21, que o butirato de hexila apresentou a maior Energia de Ativação Viscosa e o acetato de etila, a menor Energia de Ativação Viscosa.

Assim, de acordo com a TAB. 21, os resultados sugerem que o butirato de hexila apresenta uma maior sensibilidade da viscosidade quanto ao aumento de temperatura, enquanto que $\mathrm{o}$ acetato de etila apresenta uma menor sensibilidade da viscosidade quanto ao aumento de temperatura, quando comparados aos outros ésteres.

Comparando os dados indicados na TAB. 3 e na TAB. 13, observa-se que para a mesma temperatura, a viscosidade do butirato de hexila é maior que a viscosidade do acetato de etila.

Dessa forma é possível associar os valores da viscosidade com a Energia de Ativação Viscosa, onde altos valores da viscosidade estão associados a altos valores da Energia de Ativação Viscosa.

A TAB. 22 relaciona os valores da Energia de Ativação Viscosa $\left(E_{a}\right)$ com a variação da viscosidade $(\Delta \eta)$.

A variação da viscosidade $(\Delta \eta)$ corresponde à diferença entre a viscosidade na temperatura de $293,15 \mathrm{~K}$ e a viscosidade na temperatura de $343,15 \mathrm{~K}$ para cada um dos ésteres.

As exceções no cálculo correspondem ao formato de etila em que a variação da viscosidade $(\Delta \eta)$ corresponde à diferença entre a viscosidade na temperatura de $293,15 \mathrm{~K}$ e a viscosidade na temperatura de $323,15 \mathrm{~K}$ e aos acetatos de cis-3hexenila e trans-2-hexenila. Nestes dois últimos, a variação da viscosidade $(\Delta \eta)$ corresponde à diferença entre a viscosidade na temperatura de $293,15 \mathrm{~K}$ e a viscosidade na temperatura de $333,15 \mathrm{~K}$

A FIG. 7 ilustra o gráfico da Energia de Ativação Viscosa $\left(E_{a}\right)$ em função da variação da viscosidade $(\Delta \eta)$.

Pode-se observar na FIG. 7, um aumento exponencial da Energia de Ativação Viscosa à medida que a variação da viscosidade aumenta. É de se esperar esta constatação, pois a Energia de Ativação Viscosa está relacionada com a temperatura e; conforme a Eq. 
1, o efeito da temperatura sobre a viscosidade é modelado por uma relação do tipo Arrhenius, que é uma relação exponencial entre a viscosidade e a temperatura.

Tabela 22

Energia de Ativação Viscosa em função da variação da viscosidade

\begin{tabular}{c|c}
\hline$E_{a}\left(\mathrm{~kJ} . \mathrm{mol}^{-1}\right)$ & $\Delta \eta(\mathrm{mPa} . \mathrm{s})$ \\
& \\
\hline 6,454 & 0,168 \\
7,669 & 0,102 \\
8,454 & 0,208 \\
9,15 & 0,253 \\
9,188 & 0,28 \\
10,177 & 0,379 \\
10,248 & 0,407 \\
10,417 & 0,374 \\
10,687 & 0,387 \\
10,776 & 0,348 \\
10,876 & 0,499 \\
11,016 & 0,539 \\
11,104 & 0,462 \\
11,383 & 0,387 \\
11,388 & 0,384 \\
11,927 & 0,521 \\
12,745 & 0,724 \\
\hline
\end{tabular}

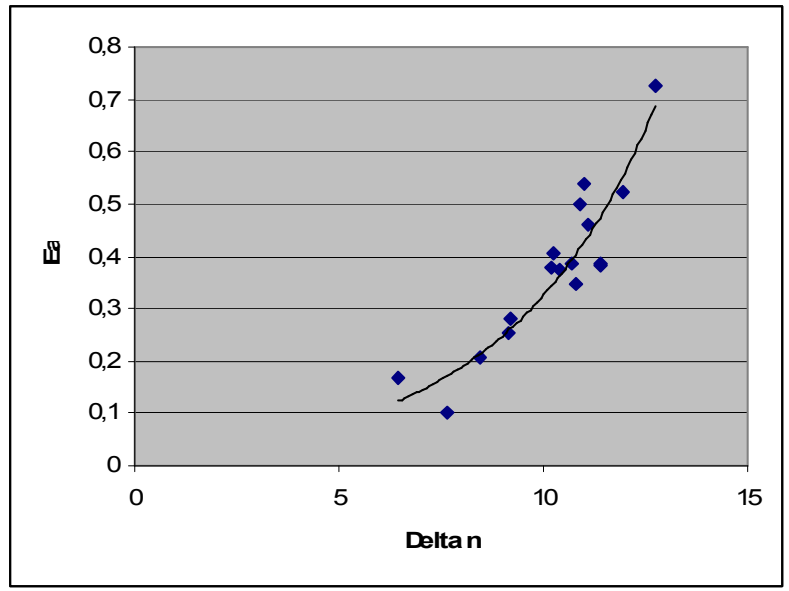

Figura 7 - Gráfico de $E_{a}$ versus $\Delta \eta$

A TAB. 23 relaciona os valores da Energia de Ativação Viscosa $\left(E_{a}\right)$ com a massa molecular (MM) de cada éster correspondente. Enquanto que a FIG. 8 ilustra o gráfico obtido pelos dados indicados nesta tabela.

Pode-se observar na FIG. 8 que a Energia de Ativação em função da massa molecular do éster apresenta um comportamento linear, de maneira que um aumento da massa molecular do éster implica em um aumento da Energia de Ativação Viscosa.

Tabela 23

Energia de Ativação Viscosa em função da massa molecular do éster

\begin{tabular}{c|c}
\hline$E_{a}\left(\mathrm{~kJ} . \mathrm{mol}^{-1}\right)$ & $\mathrm{MM}$ (u.m.a.) \\
& \\
\hline 6,454 & 74,08 \\
7,669 & 88,11 \\
8,454 & 102,13 \\
9,15 & 116,16 \\
9,188 & 116,16 \\
10,177 & 128,17 \\
10,248 & 130,19 \\
10,417 & 130,19 \\
10,687 & 130,19 \\
10,776 & 142,2 \\
10,876 & 142,2 \\
11,016 & 144,21 \\
11,104 & 144,21 \\
11,383 & 144,21 \\
11,388 & 158,23 \\
11,927 & 170,25 \\
12,745 & 172,27 \\
\hline
\end{tabular}

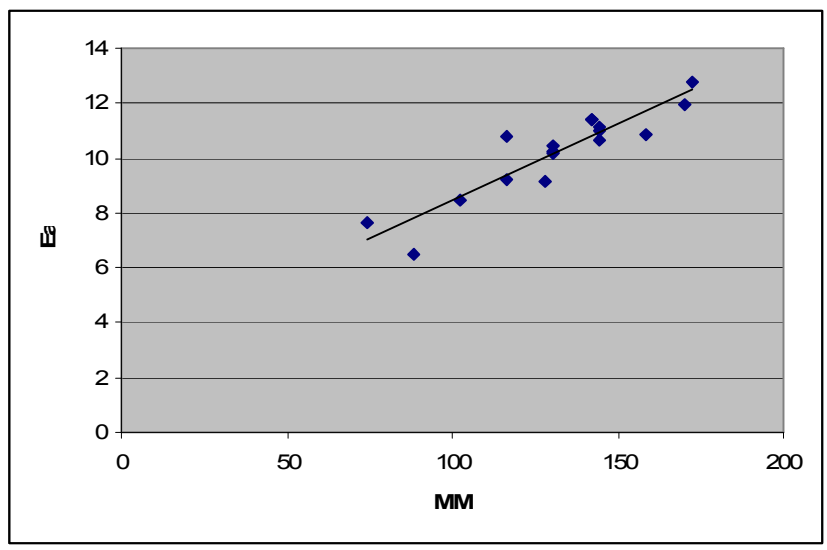

Figura 8-Gráfico de $E_{a}$ versus a massa molecular do éster 
O coeficiente de correlação $\left(r^{2}\right)$ para a análise de regressão linear dos dados de $E_{a}$ versus a massa molecular do éster forneceu como resultado um valor de 0,831 .

Segundo Callegari-Jaques (2003), citado por Lira (2004), para valores do coeficiente de correlação $\left(r^{2}\right)$ maiores ou iguais a 0,60 e menores que 0,90, a correlação linear é classificada como forte.

Dessa forma, existe uma forte correlação linear entre a Energia de Ativação Viscosa e a massa molecular do éster.

A TAB. 24 relaciona os valores da variação da viscosidade $(\Delta \eta)$ com a massa molecular do éster correspondente (MM).

A FIG. 9 ilustra o gráfico da variação da viscosidade em função da massa molecular do éster.

O coeficiente de correlação $\left(r^{2}\right)$ para a análise de regressão linear dos dados de $\Delta \eta$ versus a massa molecular do éster forneceu como resultado um valor de 0,847 .

Dessa forma, existe uma forte correlação linear entre a variação da viscosidade e a massa molecular do éster.

Quanto a valores experimentais da Energia de Ativação Viscosa de ésteres, não foram encontrados valores para esta grandeza na literatura.

A título de comparação, Granjeiro e colaboradores (2007) comentam que a Energia de Ativação Viscosa para polpas concentradas de figo-da Índia varia de 3,04 a $10,64 \mathrm{~kJ} \cdot \mathrm{mol}^{-1}$.

Canciam (2008) comenta que as Energias de Ativação Viscosa para soluções aquosas de sacarose (20, 40 e $60 \%$ em massa de sacarose) apresentaram valores, respectivamente, iguais a $17,516 \mathrm{~kJ}_{\mathrm{mol}}{ }^{-1}$, $22,714 \mathrm{~kJ} . \mathrm{mol}^{-1}$ e $34,802 \mathrm{~kJ} . \mathrm{mol}^{-1}$.
Tabela 24

Variação da viscosidade em função da massa molecular do éster correspondente

\begin{tabular}{c|c}
\hline$\Delta \eta$ (mPa.s) & MM (u.m.a.) \\
\hline 0,102 & 74,08 \\
0,168 & 88,11 \\
0,208 & 102,13 \\
0,28 & 116,16 \\
0,348 & 116,16 \\
0,253 & 128,17 \\
0,407 & 130,19 \\
0,374 & 130,19 \\
0,379 & 130,19 \\
0,387 & 142,2 \\
0,384 & 142,2 \\
0,462 & 144,21 \\
0,539 & 144,21 \\
0,387 & 144,21 \\
0,499 & 158,23 \\
0,521 & 170,25 \\
0,724 & 172,27 \\
\hline
\end{tabular}

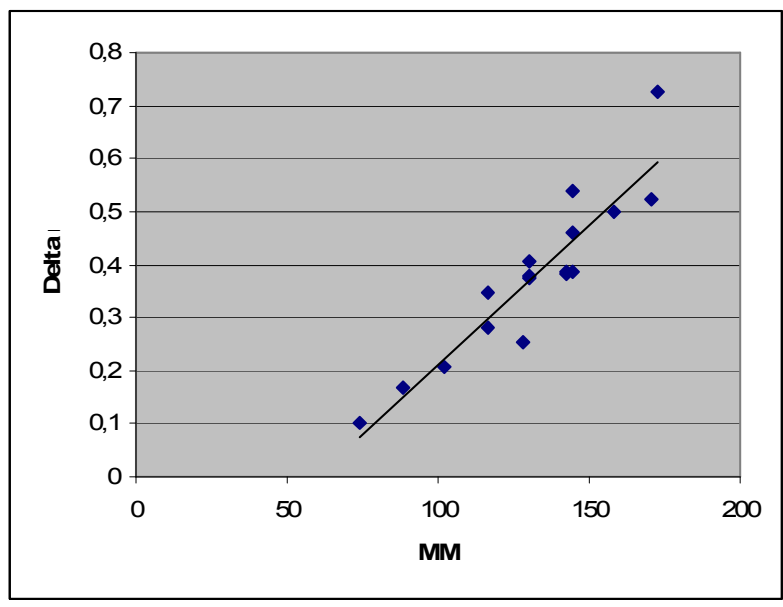

Figura 9 - Gráfico de $\Delta \eta$ versus a massa molecular do éster

Bezerra e colaboradores (2009) relatam que a Energia de Ativação Viscosa para polpa integral de morango varia de 10,907 a $14,274 \mathrm{~kJ} . \mathrm{mol}^{-1}$. Os mesmos autores comentam que a polpa integral de morango apresenta Energia de Ativação Viscosa intermediária, quando comparada a produtos alimentícios muito fluidos e muito pastosos. 
A ausência de valores da Energia de Ativação Viscosa para a maioria das substâncias químicas dificulta a comparação e a classificação para os ésteres estudados neste trabalho.

\section{Conclusão}

A Energia de Ativação Viscosa para os ésteres estudados variou de $6,454 \mathrm{~kJ} \mathrm{~mol}^{-1}$ (acetato de etila) a $12,745 \mathrm{kJmol}^{-1}$ (butirato de hexila). Sugerindo que o butirato de hexila apresenta uma maior sensibilidade da viscosidade quanto ao aumento de temperatura, quando comparado aos outros ésteres.

Para todos os ésteres, as correlações lineares foram classificadas como muito fortes.
Foi observado que existe uma forte correlação linear entre a Energia de Ativação Viscosa e a massa molecular do éster, de maneira que, à medida que se aumenta a massa molecular do éster, maior é a Energia de Ativação Viscosa.

Foram observadas também uma correlação linear forte entre variação da viscosidade e a massa molecular do éster e uma correlação exponencial entre a Energia de Ativação Viscosa e a variação da viscosidade. Em ambos os casos, a correlação foi positiva, ou seja, o aumento da variável independente implica no aumento da variável dependente.

A ausência na literatura de valores da Energia de Ativação Viscosa para os ésteres estudados neste trabalho dificulta a comparação e a classificação dos valores da Energia de Ativação Viscosa em alto, intermediário e baixo.

\section{REFERÊNCIAS}

BEZERRA, J. R. M. V.; RIGO, M.; DEMCZUK JUNIOR, B.; CÓRDOVA, K. R. V. Estudo do efeito da temperatura nas propriedades reológicas da polpa de morango (Fragaria ananassa). Revista Ambiência. Guarapuava, 2009. 11 p.

BRUICE, P. Y. Química Orgânica- volume 2 . São Paulo: Editora Pearson Education do Brasil, 2006. 704 p. ISBN: 978-85-760-5068-1.

CAMPOS, M. M; AMARAL, L. F. P.; SEOUD, O. E.; ALVARENGA, M. A.; VICENTINI, G.; YOSHIDA, M.; LOPES FILHO, J. C.; DE' ÁCQUA, A. Fundamentos de Química Orgânica. São Paulo: Editora Edgard Blücher, 1997. 640 p. ISBN: 852-120-124-9.

CANCIAM, C. A. Efeito da temperatura na viscosidade de soluções de sacarose. In: SANTOS JÚNIOR, G.; ALMEIDA, D. M.; MICHALOSKI, A. O. (eds). Série em Ciência e Tecnologia de Alimentos: agroindústria, energia e meio ambiente. Ponta Grossa: UTFPR, 2008. ISBN: 978-85-7014-041-8.

COSTA, T. S.; ORNELAS, D. L.; GUIMARÃES, P. I. C.; MERÇON, F. Confirmando a esterificação de Fischer por meio dos aromas. Revista Química Nova na Escola. São Paulo, SBQ, 2004. 3 p.
DJOJOPUTRO, H.; ISMADJI, S. Density and viscosity correlation for several common fragrance and flavor esters. Journal of Chemical and Engineering Data. Washington, 2005. 5.p.

GARCIA, V. Subproduto de destilaria de óleo fúsel: caracterização da composição química e estudo de sua aplicação industrial. 98 p. Disssertação (Mestrado em Engenharia de Processos Químicos e Bioquímicos), IMT, São Caetano do Sul, 2008.

GIAP, S. G. E. The hidden property of Arrhenius-type relationship: viscosity as a function of temperature. Journal of Physical Science. Kelantan, 2010. 11 p.

GRANJEIRO, A. A.; QUEIROZ, A. J. M.; FIGUEIRÊDO, R. M. F.; MATA, M. E. R. M. C. Viscosidades de polpas concentradas de figo-da-Índia. Revista Brasileira de Agrociência. Pelotas, 2007. 6 p.

GRATÃO, A. C. A.; BERTO, M. I.; SILVEIRA JÚNIOR, $\mathrm{V}$. Reologia do açúcar líquido invertido: influência da temperatura na viscosidade. Revista Ciência e Tecnologia de Alimentos. Campinas, 2004. 5 p.

LIN, C. F.; HSIEH, H. M.; LEE, L.S . Estimations of the viscosities of binary mixtures with different equations 
of state and mixing rules. Journal of the Chinese Institute of Chemical Engineers. Taiwan, 2007, 20 p.

LIRA, S. A. Análise de correlação: abordagem teórica e de construção dos coeficientes com aplicação. 196 p. Dissertação (Mestrado em Ciências), UFPR, Curitiba, 2004.

OLIVEIRA, R. C.; BARROS, S. T. D.; ROSSI, R. M. Aplicação da metodologia Bayesiana para o estudo reológico da polpa de uva. Revista Brasileira de Produtos Agroindustriais. Campina Grande, 2009. 8 p.

PEREIRA, E. A.; QUEIROZ, A. J. M.; FIGUEIRÊDO, R. M. F. Comportamento reológico de mel da abelha uruçu (Melipona scutellaris, L.). Revista Ciências Exatas e Naturais. Guarapuava, 2003. 8 p.

RIBEIRO, L. D.; DA CRUZ, S. R. A.; REIS, R. A. Cálculo de viscosidade de misturas não eletrolíticas. In: Congresso Brasileiro de Engenharia Química em Iniciação Científica, 6 $6^{\circ}$ Campinas. Anais. Unicamp, 2005.

SHAMES, I. H. Mecânica dos Fluidos - volume 1. São Paulo: Editora Edgard Blücher, 1999. 192 p. ISBN: 852-120-170-2.
SILVA, A. A.; PAREDES, M. L. L.; REIS, R. A. Modelagem de viscosidade de misturas não eletrolíticas. In: Congresso Brasileiro de Engenharia Química em Iniciação Científica, $7^{\circ}$, São Carlos. Anais. UFSCAR, 2007.

SILVA, S. A. Estudo do comportamento reológico dos adesivos hot melt PSA e a sua relação com a composição e as propriedades adesivas. $123 \mathrm{p}$. Dissertação (Mestrado em Engenharia Química), UFRGS, Porto Alegre, 2008.

SKORONSKI, E. Estudo cinético da síntese do octanoato de n-pentila catalisada pela enzima Lipozyme TL IM. 87 p. Dissertação (Mestrado em Engenharia Química), UFSC, Florianópolis, 2006.

SOLOMONS, T. W. G.; FRYHLE, C. B. Química Orgânica- volume 2. Rio de Janeiro: LTC Editora, 2009. 518 p. ISBN: 852-16-1678-3.

TRIOLA, M .F. Introdução à Estatística. Rio de Janeiro: LTC Editora, 2008. 720 p. ISBN: 978-852161-586-6. 